\title{
CONVERGENCE RATE OF EXTREMES FOR THE GENERAL ERROR DISTRIBUTION
}

\author{
PENG ZUOXIANG, ${ }^{*}$ Southwest University \\ SARALEES NADARAJAH, ${ }^{* *}$ University of Manchester \\ LIN FUMING, ${ }^{* * *}$ Sichuan University of Science and Engineering
}

\begin{abstract}
Let $\left\{X_{n}, n \geq 1\right\}$ be an independent, identically distributed random sequence with each $X_{n}$ having the general error distribution. In this paper we derive the exact uniform convergence rate of the distribution of the maximum to its extreme value limit.
\end{abstract}

Keywords: Extreme value distribution; general error distribution; maximum; uniform convergence rate

2010 Mathematics Subject Classification: Primary 62E20

Secondary 60F05

\section{Introduction}

Let $\left\{X_{n}, n \geq 1\right\}$ be a sequence of independent, identically distributed random variables with common distribution function $F(x)$. Let $M_{n}=\max _{1 \leq k \leq n} X_{k}$ denote the partial maximum of $\left\{X_{n}, n \geq 1\right\}$. Suppose that there exist constants $a_{n}>0$ and $b_{n} \in \mathbb{R}$, and a nondegenerate distribution $G(x)$ such that

$$
\lim _{n \rightarrow \infty} \mathrm{P}\left(M_{n} \leq a_{n} x+b_{n}\right)=\lim _{n \rightarrow \infty} F^{n}\left(a_{n} x+b_{n}\right)=G(x)
$$

for all continuity points of $G$. Then $G$ must belong to one of the following three classes:

$$
\begin{aligned}
& \text { Class I (Gumbel) : } \quad \Lambda(x)=\exp \left\{-\mathrm{e}^{-x}\right\}, \quad x \in \mathbb{R} \text {; } \\
& \text { Class II (Fréchet) : } \quad \Phi_{\alpha}(x)=\left\{\begin{array}{ll}
0 & \text { if } x<0, \\
\exp \left\{-x^{-\alpha}\right\} & \text { if } x \geq 0,
\end{array} \text { for some } \alpha>0\right. \text {; } \\
& \text { Class III (Weibull) : } \quad \Psi_{\alpha}(x)=\left\{\begin{array}{ll}
\exp \left\{-(-x)^{\alpha}\right\} & \text { if } x<0, \\
1 & \text { if } x \geq 0,
\end{array} \text { for some } \alpha>0 .\right.
\end{aligned}
$$

We say that $F$ is in the domain of attraction of $G$ if (1.1) holds. We denote such a fact by $F \in D(G)$. Criteria for $F \in D(G)$ and the choice of norming constants, $a_{n}$ and $b_{n}$, can be found in Leadbetter et al. (1983) and Resnick (1987).

One interesting problem in extreme value theory is the convergence rate of $F^{n}\left(a_{n} x+b_{n}\right)$ to any one of the extreme value distributions. There are penultimate and ultimate approximations.

\footnotetext{
Received 14 April 2009; revision received 8 April 2010.

* Postal address: School of Mathematics and Statistics, Southwest University, Chongqing, 400715, P. R. China.

** Postal address: School of Mathematics, University of Manchester, Oxford Road, Manchester, M13 9PL, UK.

Email address: mbbsssn2@manchester.ac.uk

*** Postal address: Department of Mathematics, Sichuan University of Science and Engineering, Zigong, Sichuan, 643000, P. R. China.
} 
For penultimate approximations of $F^{n}\left(a_{n} x+b_{n}\right)$, see Anderson (1971), Cohen (1982b), Gomes (1984), Gomes and de Haan (1999), Kaufmann (2000), and Reiss (1989). For the uniform convergence rate of $F^{n}\left(a_{n} x+b_{n}\right)$ to its extreme value limit, $\Lambda(x)$, Hall and Wellner (1979) showed that the convergence rate is proportional to $1 / n$ if $F$ is exponential. For the normal distribution, Hall (1979) proved the following result:

$$
\frac{c_{1}}{\log n}<\sup _{x \in \mathbb{R}}\left|\Phi^{n}\left(a_{n} x+b_{n}\right)-\Lambda(x)\right|<\frac{c_{2}}{\log n}
$$

for $n>n_{0}$, where constants $0<c_{1}<c_{2}, \Phi(x)$ denotes the normal distribution function, and the norming constants $a_{n}$ and $b_{n}$ are given by

$$
2 \pi b_{n}^{2} \exp \left\{b_{n}^{2}\right\}=n^{2}, \quad a_{n}=b_{n}^{-1} .
$$

Hall (1979) showed that $1 / \log n$ is the best convergence rate for the maxima of normal random variables. Castro (1987) proved a similar result for the gamma distribution. For related work on the uniform convergence rate of extremes, see Smith (1982), Cohen (1982a), Falk (1986), and Kaufmann (1995). For work using second-order conditions, see Balkema and de Haan (1990) and de Haan and Resnick (1996). For the rate of convergence of intermediate order statistics, see Cheng et al. (1997). For the convergence rate of the maximum of stationary normal sequences, see Rootzén (1983).

Our interest in this paper is to consider the uniform convergence rate of (1.1) when $X_{n}$ follows the general error distribution (GED). The GED being a generalization of the normal distribution is one of the most widely applied (if not the most applied) distributions in statistics. The probability density function of the GED is given by

$$
F^{\prime}(x)=\frac{v \exp \left\{-(1 / 2)|x / \lambda|^{v}\right\}}{\lambda 2^{1+1 / v} \Gamma(1 / v)}, \quad x \in \mathbb{R},
$$

with parameter $v>0$, where $\lambda=\left(2^{-2 / v} \Gamma(1 / v) / \Gamma(3 / v)\right)^{1 / 2}$ and $\Gamma(\cdot)$ denotes the gamma function (cf. Nelson (1991, p. 352)). The GED is standard normal if $v=2$. Peng et al. (2009) studied the tail behavior of the GED and the limiting behavior of its partial maximum. In order to obtain the uniform convergence rate of extremes from the GED, we cite some results from Peng et al. (2009).

In the sequel, let $\left\{X_{n}, n \geq 1\right\}$ be a sequence of independent, identically distributed random variables with common distribution $F \sim \operatorname{GED}(v)$. As before, let $M_{n}$ denote the partial maximum of $\left\{X_{n}, n \geq 1\right\}$. Peng et al. (2009) proved that

$$
\lim _{n \rightarrow \infty} \mathrm{P}\left(M_{n} \leq \alpha_{n} x+\beta_{n}\right)=\lim _{n \rightarrow \infty} F^{n}\left(\alpha_{n} x+\beta_{n}\right)=\Lambda(x)
$$

for $v>1$ and all $x \in \mathbb{R}$, where

$$
\alpha_{n}=\frac{2^{1 / v} \lambda}{v(\log n)^{1-1 / v}}
$$

and

$$
\beta_{n}=2^{1 / v} \lambda(\log n)^{1 / v}-\frac{2^{1 / v} \lambda[((v-1) / v) \log \log n+\log \{2 \Gamma(1 / v)\}]}{v(\log n)^{1-1 / v}} .
$$

It follows from Peng et al. (2009) that

$$
1-F(x)=c(x) \exp \left\{-\int_{\lambda}^{x} \frac{g(t)}{f(t)} \mathrm{d} t\right\}
$$


for $v>1$ and sufficiently large $x$, where

$$
c(x) \rightarrow \frac{\exp \{-1 / 2\}}{2^{1 / v} \Gamma(1 / v)} \quad \text { as } x \rightarrow \infty, \quad f(t)=2 v^{-1} \lambda^{v} t^{1-v},
$$

and

$$
g(t)=1+2(v-1) v^{-1} \lambda^{v} t^{-v} .
$$

Noting that $f^{\prime}(t) \rightarrow 0$ and $g(t) \rightarrow 1$ as $t \rightarrow \infty$, and by Proposition 1.1(a) and Corollary 1.7 of Resnick (1987), we can choose the norming constants $a_{n}$ and $b_{n}$ in such a way that $b_{n}$ is the solution of the equation

$$
2^{1 / v} \lambda^{1-v} \Gamma\left(\frac{1}{v}\right) b_{n}^{v-1} \exp \left\{\frac{b_{n}^{v}}{2 \lambda^{v}}\right\}=n
$$

with

$$
a_{n}=f\left(b_{n}\right)=2 v^{-1} \lambda^{v} b_{n}^{1-v} .
$$

Note that, for the normal distribution, $\lambda=1,(1.5)$ and (1.6) reduce to (1.2). We prove that the best uniform convergence rate of $F^{n}\left(a_{n} x+b_{n}\right)$ to its extreme value limit is proportional to $1 / \log n$. However, for $F^{n}\left(\alpha_{n} x+\beta_{n}\right)$, the convergence rate is no better than $(\log \log n)^{2} / \log n$ even though $\alpha_{n} / a_{n} \rightarrow 1$ and $\left(\beta_{n}-b_{n}\right) / a_{n} \rightarrow 0$ as $n \rightarrow \infty$.

This paper is organized as follows. In Section 2 we provide the main results, with their proofs deferred to Section 4. Some auxiliary results are given in Section 3.

\section{Main results}

We provide two main results. Theorem 2.1 shows that the uniform convergence rate of $F^{n}\left(a_{n} x+b_{n}\right)$ to its limit is of the order of $O(1 / \log n)$. Theorem 2.2 shows that the pointwise convergence rate of $F^{n}\left(\alpha_{n} x+\beta_{n}\right)$ to its limit is of the order of $O\left((\log \log n)^{2} / \log n\right)$.

Theorem 2.1. Let $\left\{X_{n}, n \geq 1\right\}$ denote a sequence of independent, identically distributed random variables with common distribution $F \sim \operatorname{GED}(v)$ and parameter $v>1$. Then there exist absolute constants $0<c_{1}(v)<c_{2}(v)$ such that

$$
\frac{c_{1}(v)}{\log n}<\sup _{x \in \mathbb{R}}\left|F^{n}\left(a_{n} x+b_{n}\right)-\Lambda(x)\right|<\frac{c_{2}(v)}{\log n}
$$

for $n \geq 2$, where $b_{n}$ and $a_{n}$ are defined by (1.5) and (1.6), respectively.

Theorem 2.2. Let $\alpha_{n}$ and $\beta_{n}$ be defined by (1.3) and (1.4), respectively. Then

$$
F^{n}\left(\alpha_{n} x+\beta_{n}\right)-\Lambda(x) \sim \exp \left\{-\mathrm{e}^{-x}\right\} \mathrm{e}^{-x} \frac{(v-1)^{3}}{2 v^{3}} \frac{(\log \log n)^{2}}{\log n}
$$

for large $n$.

\section{Auxiliary results}

We will use the following properties of the GED distribution (cf. Equations (6) and (7) of Hall (1979, p. 434)). 
Proposition 3.1. Let $F$ denote the distribution function of $\operatorname{GED}(v)$ with $v>1$. For $x>0$, we have

$$
\begin{aligned}
1-F(x) & =\frac{2^{-1 / v} \lambda^{v-1}}{\Gamma(1 / v)} x^{1-v} \exp \left\{-\frac{x^{v}}{2 \lambda^{v}}\right\}-r_{v}(x) \\
& =\frac{2^{-1 / v} \lambda^{v-1}}{\Gamma(1 / v)} x^{1-v} \exp \left\{-\frac{x^{v}}{2 \lambda^{v}}\right\}\left(1-\frac{2(v-1)}{v} \lambda^{v} x^{-v}\right)+s_{v}(x),
\end{aligned}
$$

where

$$
0<r_{v}(x)<\frac{2^{1-1 / v} \lambda^{2 v-1}(v-1)}{v \Gamma(1 / v)} x^{1-2 v} \exp \left\{-\frac{x^{v}}{2 \lambda^{v}}\right\}
$$

and

$$
0<s_{v}(x)<\frac{2^{2-1 / v} \lambda^{3 v-1}(v-1)(2 v-1)}{v^{2} \Gamma(1 / v)} x^{1-3 v} \exp \left\{-\frac{x^{v}}{2 \lambda^{v}}\right\} .
$$

Proof. By integration by parts we have

$$
\begin{aligned}
1-F(x) & =\frac{2^{-1 / v} \lambda^{v-1}}{\Gamma(1 / v)} x^{1-v} \exp \left\{-\frac{x^{v}}{2 \lambda^{v}}\right\}-\frac{2^{-1 / v} \lambda^{v-1}(v-1)}{\Gamma(1 / v)} \int_{x}^{\infty} t^{-v} \exp \left\{-\frac{t^{v}}{2 \lambda^{v}}\right\} \mathrm{d} t \\
& =: \frac{2^{-1 / v} \lambda^{v-1}}{\Gamma(1 / v)} x^{1-v} \exp \left\{-\frac{x^{v}}{2 \lambda^{v}}\right\}-r_{v}(x),
\end{aligned}
$$

which is (3.1). Similarly,

$$
r_{v}(x)=\frac{2^{1-1 / v} \lambda^{2 v-1}(v-1)}{v \Gamma(1 / v)} x^{1-2 v} \exp \left\{-\frac{x^{v}}{2 \lambda^{v}}\right\}-s_{v}(x) .
$$

Substituting this into (3.1), we obtain (3.2), where

$$
\begin{aligned}
s_{v}(x)= & \frac{2^{1-1 / v} \lambda^{2 v-1}(v-1)(2 v-1)}{v \Gamma(1 / v)} \int_{x}^{\infty} t^{-2 v} \exp \left\{-\frac{t^{v}}{2 \lambda^{v}}\right\} \mathrm{d} t \\
= & \frac{2^{2-1 / v} \lambda^{3 v-1}(v-1)(2 v-1)}{v^{2} \Gamma(1 / v)} x^{1-3 v} \exp \left\{-\frac{x^{v}}{2 \lambda^{v}}\right\} \\
& -\frac{2^{2-1 / v} \lambda^{3 v-1}(v-1)(2 v-1)(3 v-1)}{v^{2} \Gamma(1 / v)} \int_{x}^{\infty} t^{-3 v} \exp \left\{-\frac{t^{v}}{2 \lambda^{v}}\right\} \mathrm{d} t \\
< & \frac{2^{2-1 / v} \lambda^{3 v-1}(v-1)(2 v-1)}{v^{2} \Gamma(1 / v)} x^{1-3 v} \exp \left\{-\frac{x^{v}}{2 \lambda^{v}}\right\} .
\end{aligned}
$$

This completes the proof.

For the norming constants $a_{n}$ and $b_{n}$ defined by (1.6) and (1.5), respectively, let

$$
a_{n}^{*}=a_{n} r_{n}, \quad b_{n}^{*}=b_{n}+\delta_{n} a_{n},
$$

where $r_{n} \rightarrow 1$ and $\delta_{n} \rightarrow 0$ as $n \rightarrow \infty$. The following expansion is needed.

Proposition 3.2. Let $a_{n}^{*}$ and $b_{n}^{*}$ be defined by (3.3). For fixed $x \in \mathbb{R}$ and sufficiently large $n$,

$$
\begin{gathered}
F^{n}\left(a_{n}^{*} x+b_{n}^{*}\right)-\Lambda(x)=\Lambda(x) \mathrm{e}^{-x}\left\{(v-1) a_{n} b_{n}^{-1}\left(1+x+\frac{x^{2}}{2}\right)+\left(r_{n}-1\right) x+\delta_{n}\right. \\
\left.+O\left[\left(a_{n} b_{n}^{-1}\right)^{2}+\left(r_{n}-1\right)^{2}+\delta_{n}^{2}\right]\right\} .
\end{gathered}
$$


Proof. Note that $b_{n} \sim 2^{1 / v} \lambda(\log n)^{1 / v}$ by (1.5), which implies that

$$
a_{n} b_{n}^{-1} \sim(\log n)^{-1} \rightarrow 0
$$

by (1.6). So, by (1.6) we have

$$
\begin{aligned}
& \frac{2^{-1 / v} \lambda^{v-1}}{\Gamma(1 / v)}\left(a_{n}^{*} x+b_{n}^{*}\right)^{1-v} \exp \left\{-\frac{\left(a_{n}^{*} x+b_{n}^{*}\right)^{v}}{2 \lambda^{v}}\right\} \\
& =n^{-1}\left[1+a_{n} b_{n}^{-1}\left(r_{n} x+\delta_{n}\right)\right]^{1-v} \exp \left\{-\frac{b_{n}^{v}}{2 \lambda^{v}}\left[\left(1+a_{n} b_{n}^{-1}\left(r_{n} x+\delta_{n}\right)\right)^{v}-1\right]\right\} \\
& =n^{-1}\left\{1-(v-1)\left[a_{n} b_{n}^{-1}\left(r_{n} x+\delta_{n}\right)\right]+\frac{v(v-1)}{2}\left[a_{n} b_{n}^{-1}\left(r_{n} x+\delta_{n}\right)\right]^{2}\right. \\
& \left.+O\left(\left[a_{n} b_{n}^{-1}\left(r_{n} x+\delta_{n}\right)\right]^{3}\right)\right\} \\
& \times \exp \left\{-x-\left(r_{n}-1\right) x-\delta_{n}-\frac{v-1}{2}\left(r_{n} x+\delta_{n}\right)^{2} a_{n} b_{n}^{-1}\right. \\
& \left.+O\left(\left(a_{n} b_{n}^{-1}\right)^{2}\left(\left(r_{n} x+\delta_{n}\right)\right)^{3}\right)\right\} \\
& =n^{-1} \mathrm{e}^{-x}\left\{1-(v-1)\left[a_{n} b_{n}^{-1}\left(r_{n} x+\delta_{n}\right)\right]+\frac{v(v-1)}{2}\left[a_{n} b_{n}^{-1}\left(r_{n} x+\delta_{n}\right)\right]^{2}\right. \\
& \left.+O\left(\left[a_{n} b_{n}^{-1}\left(r_{n} x+\delta_{n}\right)\right]^{3}\right)\right\} \\
& \times\left\{1-\left(r_{n}-1\right) x-\delta_{n}-\frac{v-1}{2}\left(r_{n} x+\delta_{n}\right)^{2} a_{n} b_{n}^{-1}+O\left(\left(a_{n} b_{n}^{-1}\right)^{2}\left(r_{n} x+\delta_{n}\right)^{3}\right)\right. \\
& +\frac{1}{2}\left(\left(r_{n}-1\right) x+\delta_{n}+\frac{v-1}{2}\left(r_{n} x+\delta_{n}\right)^{2} a_{n} b_{n}^{-1}\right)^{2} \\
& \left.+O\left(\left(a_{n} b_{n}^{-1}\right)^{3}+\left(r_{n}-1\right)^{3}+\delta_{n}^{3}\right)\right\} \\
& =n^{-1} \mathrm{e}^{-x}\left\{1-\left(r_{n}-1\right) x-\delta_{n}-(v-1) a_{n} b_{n}^{-1}\left(x+\frac{1}{2} x^{2}\right)\right. \\
& \left.+O\left(\left(a_{n} b_{n}^{-1}\right)^{2}+\left(r_{n}-1\right)^{2}+\delta_{n}^{2}\right)\right\} \text {. }
\end{aligned}
$$

Similarly,

$$
\left(a_{n}^{*} x+b_{n}^{*}\right)^{-v}=\frac{a_{n} b_{n}^{-1}}{2 v^{-1} \lambda^{v}}-\frac{\left(a_{n} b_{n}^{-1}\right)^{2}}{2 v^{-2} \lambda^{v}}\left(r_{n} x+\delta_{n}\right)+O\left(\left(a_{n} b_{n}^{-1}\right)^{3}\right) .
$$

By (3.4) and (3.5), we obtain

$$
\left(a_{n}^{*} x+b_{n}^{*}\right)^{1-3 v} \exp \left\{-\frac{\left(a_{n}^{*} x+b_{n}^{*}\right)^{v}}{2 \lambda^{v}}\right\}=O\left(n^{-1}\left(a_{n} b_{n}^{-1}\right)^{2}\right) .
$$

So,

$$
s_{v}\left(a_{n}^{*} x+b_{n}^{*}\right)=O\left(n^{-1}\left(a_{n} b_{n}^{-1}\right)^{2}\right),
$$

where $s_{v}(x)$ is defined as in Proposition 3.1. So, by (3.2), (3.4), and (3.6), we have

$$
\begin{aligned}
1-F\left(a_{n}^{*} x+b_{n}^{*}\right)=n^{-1} \mathrm{e}^{-x} & \left\{1-\left(r_{n}-1\right) x-\delta_{n}-(v-1) a_{n} b_{n}^{-1}\left(1+x+\frac{1}{2} x^{2}\right)\right. \\
& \left.+O\left[\left(a_{n} b_{n}^{-1}\right)^{2}+\left(r_{n}-1\right)^{2}+\delta_{n}^{2}\right]\right\}
\end{aligned}
$$


for large $n$. So,

$$
\begin{aligned}
& F^{n}\left(a_{n}^{*} x+b_{n}^{*}\right)-\Lambda(x)=\left\{1-n^{-1} \mathrm{e}^{-x}\left[1-\left(r_{n}-1\right) x-\delta_{n}-(v-1) a_{n} b_{n}^{-1}\left(1+x+\frac{1}{2} x^{2}\right)\right.\right. \\
& \left.\left.+O\left[\left(a_{n} b_{n}^{-1}\right)^{2}+\left(r_{n}-1\right)^{2}+\delta_{n}^{2}\right]\right]\right\}^{n}-\Lambda(x) \\
& =\Lambda(x) \mathrm{e}^{-x}\left\{(v-1) a_{n} b_{n}^{-1}\left(1+x+\frac{x^{2}}{2}\right)+\left(r_{n}-1\right) x+\delta_{n}\right. \\
& \left.+O\left[\left(a_{n} b_{n}^{-1}\right)^{2}+\left(r_{n}-1\right)^{2}+\delta_{n}^{2}\right]\right\},
\end{aligned}
$$

which completes the proof.

\section{The proofs}

We first prove Theorem 2.2 as it is relatively easy.

Proof of Theorem 2.2. Firstly, we derive the following asymptotic expansions of $b_{n}$ defined by (1.5):

$$
b_{n}=\beta_{n}+o\left((\log n)^{1 / v-1}\right),
$$

and

$$
b_{n}=\beta_{n}-\frac{2^{1 / v-1} \lambda(v-1)}{v^{2}} \frac{B_{n}^{2}-2 B_{n}}{(\log n)^{2-1 / v}}+O\left(\frac{B_{n}^{2}}{(\log n)^{3-1 / v}}\right) \text {, }
$$

where

$$
B_{n}=\frac{v-1}{v} \log \log n+\log 2 \Gamma\left(\frac{1}{v}\right)
$$

and $\beta_{n}$ is defined by (1.4). By Corollary 1.7 of Resnick (1987) we have

$$
\mathrm{P}\left(M_{n} \leq a_{n} x+b_{n}\right) \rightarrow \Lambda(x) .
$$

By arguments similar to those used in Example 2 of Resnick (1987, pp. 71-72), we can obtain (4.1). Now set

$$
b_{n}=\beta_{n}+\theta_{n},
$$

where $\theta_{n}=o\left((\log n)^{1 / v-1}\right)$. Note that

$$
\log (1-x)=-x+\frac{1}{2} x^{2}+O\left(x^{3}\right) \quad \text { as } x \rightarrow 0
$$

and that

$$
(1-x)^{v}=1-v x+\frac{v(v-1)}{2} x^{2}+O\left(x^{3}\right) \quad \text { as } x \rightarrow 0 .
$$

Substituting $b_{n}=\beta_{n}+\theta_{n}$ into

$$
\log 2^{1 / v} \lambda^{1-v} \Gamma\left(\frac{1}{v}\right)+(v-1) \log b_{n}+\frac{b_{n}^{v}}{2 \lambda^{v}}=\log n,
$$

we can obtain

$$
\begin{aligned}
& \left(\frac{v-1}{2^{1 / v} \lambda \log n}-\frac{B_{n}}{2^{1 / v} v \lambda(\log n)^{2}}+\frac{v}{2^{1 / v} \lambda}-\frac{v-1}{2^{1 / v} \lambda} \frac{B_{n}}{\log n}\right) \frac{\theta_{n}}{(\log n)^{1 / v-1}} \\
& +\left(\frac{1}{2^{2 / v+1} \lambda^{2}(\log n)^{2}}+\frac{v(v-1)}{2^{1+2 / v} \lambda \log n}\right) \frac{\theta_{n}^{2}}{(\log n)^{2 / v-2}} \\
& =\frac{v-1}{v \log n}\left(B_{n}-\frac{B_{n}^{2}}{2}\right)+O\left(\frac{(\log \log n)^{3}}{\log ^{2} n}\right)
\end{aligned}
$$


from which we can derive

$$
\theta_{n} \sim-\frac{2^{1 / v-1} \lambda(v-1)}{v^{2}} \frac{B_{n}^{2}-2 B_{n}}{(\log n)^{2-1 / v}}
$$

Once again, let

$$
\theta_{n}=-\frac{2^{1 / v-1} \lambda(v-1)}{v^{2}} \frac{B_{n}^{2}-2 B_{n}}{(\log n)^{2-1 / v}}+\vartheta_{n},
$$

where $\vartheta_{n}=o\left((\log \log n)^{2} /(\log n)^{2-1 / v}\right)$. By similar arguments, we can obtain (4.2). Note that

$$
\begin{gathered}
a_{n} b_{n}^{-1} \sim \frac{1}{v \log n}, \quad r_{n}-1=\frac{a_{n}^{*}}{a_{n}}-1 \sim-\frac{(v-1)^{2}}{v^{2}} \frac{\log \log n}{\log n}, \\
\delta_{n}=\frac{b_{n}^{*}-b_{n}}{a_{n}} \sim \frac{(v-1)^{3}}{2 v^{3}} \frac{(\log \log n)^{2}}{\log n},
\end{gathered}
$$

for large $n$. So, the result follows by Proposition 3.2.

Proof of Theorem 2.1. Letting $r_{n}=1, \delta_{n}=0$ in (3.3), and noting that $a_{n} b_{n}^{-1} \sim 1 / \log n$, by Proposition 3.2 we can prove that there exists an absolute constant $c_{1}>0$ such that

$$
\sup _{x \in \mathbb{R}}\left|F^{n}\left(a_{n} x+b_{n}\right)-\Lambda(x)\right|>\frac{c_{1}}{\log n}
$$

for $n \geq 2$. In order to obtain the upper bound, we need to prove that

$$
\begin{gathered}
\sup _{0 \leq x<\infty}\left|F^{n}\left(a_{n} x+b_{n}\right)-\Lambda(x)\right|<d_{1} a_{n} b_{n}^{-1}, \\
\sup _{-c_{n} \leq x<0}\left|F^{n}\left(a_{n} x+b_{n}\right)-\Lambda(x)\right|<d_{2} a_{n} b_{n}^{-1}, \\
\sup _{-\infty<x \leq-c_{n}}\left|F^{n}\left(a_{n} x+b_{n}\right)-\Lambda(x)\right|<d_{3} a_{n} b_{n}^{-1},
\end{gathered}
$$

for $n \geq 2$, where $d_{i}=d_{i}(v)>0, i=1,2,3$, are absolute constants and $c_{n}=: \log \log b_{n}^{v}$ is positive for $n \geq 2$. Note that, from (4.1),

$$
0.8 \lambda^{v} \log n<b_{n}^{v}<2 \lambda^{v} \log n
$$

and

$$
\sup _{n \geq 2} \frac{1}{b_{n}^{v}} \log \lg b_{n}^{v}<\sup _{n \geq 2} \frac{\log \log \left(2 \lambda^{v} \log n\right)}{0.8 \lambda^{v} \log n}<\frac{v}{2 \lambda^{v}}
$$

for $n>2$. So, $b_{n}-a_{n} c_{n}>0$ for $n>2$.

Firstly, consider the case in which $x \geq-c_{n}$. Let

$$
\begin{gathered}
R_{n}=-\left[n \log F\left(a_{n} x+b_{n}\right)+n \Psi_{n}(x)\right], \quad B_{n}(x)=\exp \left\{-R_{n}\right\}, \\
A_{n}(x)=\exp \left\{-n \Psi_{n}(x)+\mathrm{e}^{-x}\right\},
\end{gathered}
$$

where $\Psi_{n}(x)=1-F\left(a_{n} x+b_{n}\right)$. Note that, for $v>1$,

$$
b_{n}^{v}-\left(b_{n}-a_{n} c_{n}\right)^{v}=\int_{b_{n}-a_{n} c_{n}}^{b_{n}} v t^{v-1} \mathrm{~d} t<v a_{n} c_{n} b_{n}^{v-1},
$$


and, by (3.1), (1.5), and (1.6), we have

$$
\begin{aligned}
\Psi_{n}(x) & <\Psi_{n}\left(-c_{n}\right) \\
& <\frac{\lambda^{v-1} 2^{-1 / v}}{\Gamma(1 / v)}\left(b_{n}-a_{n} c_{n}\right)^{1-v} \exp \left\{-\frac{\left(b_{n}-a_{n} c_{n}\right)^{v}}{2 \lambda^{v}}\right\} \\
& =n^{-1}\left(1-b_{n}^{-1} a_{n} c_{n}\right)^{1-v} \exp \left\{-\left(\frac{\left(b_{n}-a_{n} c_{n}\right)^{v}}{2 \lambda^{v}}-\frac{b_{n}^{v}}{2 \lambda^{v}}\right)\right\} \\
& <n^{-1}\left(1-b_{n}^{-1} a_{n} c_{n}\right)^{1-v} \exp \left\{\frac{v a_{n} b_{n}^{v-1} c_{n}}{2 \lambda^{v}}\right\} \\
& =n^{-1}\left(1-b_{n}^{-1} a_{n} \log \log b_{n}^{v}\right)^{1-v} \exp \left\{\frac{v a_{n} b_{n}^{v-1} \log \log b_{n}^{v}}{2 \lambda^{v}}\right\} \\
& =\left(1-\frac{2 \lambda^{v}}{v} b_{n}^{-v} \log \log b_{n}^{v}\right)^{1-v} n^{-1} \exp \left\{\log \log b_{n}^{v}\right\} \\
& <\sup _{n \geq 2}\left\{\left(1-\frac{2 \lambda^{v}}{v} b_{n}^{-v} \log \log b_{n}^{v}\right)^{1-v} n^{-1} \log \left(2 \lambda^{v} \log n\right)\right\} \\
& =\mathfrak{C}_{1}(v) \\
& <1 .
\end{aligned}
$$

So,

$$
\inf _{x \geq-c_{n}}\left(1-\Psi_{n}(x)\right)>1-\mathfrak{C}_{1}(v)>0 .
$$

Noting that

$$
\log (1-x)<-x, \quad \log (1-x)>-x-\frac{x^{2}}{2(1-x)} \quad \text { for } 0<x<1,
$$

we have

$$
\begin{aligned}
0<R_{n}(x) & \leq \frac{n \Psi_{n}^{2}(x)}{2\left[1-\Psi_{n}(x)\right]} \\
& <\frac{n \Psi_{n}^{2}\left(-c_{n}\right)}{2\left[1-\Psi_{n}\left(-c_{n}\right)\right]} \\
& <\frac{n^{-1}\left(1-a_{n} b_{n}^{-1} c_{n}\right)^{2-2 v} a_{n}^{-1} b_{n}\left(\exp \left\{v a_{n} b_{n}^{v-1} c_{n} / 2 \lambda^{v}\right\}\right)^{2}}{2\left[1-\Psi_{n}\left(-c_{n}\right)\right] a_{n}^{-1} b_{n}} \\
& <\left(\frac{2 \mathfrak{C}_{1}(v)}{\log \left(2 \lambda^{v} \log 2\right)}\right)^{2} \frac{v}{4 \lambda^{v}\left(1-\mathfrak{C}_{1}(v)\right)} \frac{n^{-1} b_{n}^{v} \exp \left\{2 c_{n}\right\}}{a_{n}^{-1} b_{n}}
\end{aligned}
$$

Noting that $0.8 \lambda^{v} \log n<b_{n}^{v}<2 \lambda^{v} \log n$ for $n>2$, we obtain

$$
n^{-1} b_{n}^{v} \exp \left\{2 c_{n}\right\}<n^{-1}(2 \lambda \log n)^{3}<\mathfrak{C}_{2}(v) \text { for } n>2 .
$$

Substituting this into (4.7), we obtain

$$
R_{n}(x)<\left(\frac{2 \mathfrak{C}_{1}(v)}{\log \left(2 \lambda^{v} \log 2\right)}\right)^{2} \frac{v \mathfrak{C}_{2}(v)}{4 \lambda^{v}\left(1-\mathfrak{C}_{1}(v)\right)} a_{n} b_{n}^{-1}=\mathfrak{C}_{3}(v) a_{n} b_{n}^{-1} .
$$


So,

$$
\left|B_{n}(x)-1\right|<R_{n}(x)<\mathfrak{C}_{3}(v) a_{n} b_{n}^{-1} \text { for } n>2 .
$$

By inequality (4.8) we have

$$
\begin{aligned}
\left|F^{n}\left(a_{n} x+b_{n}\right)-\Lambda(x)\right| & \leq \Lambda(x) B_{n}(x)\left|A_{n}(x)-1\right|+\left|B_{n}(x)-1\right| \\
& <\Lambda(x)\left|A_{n}(x)-1\right|+\mathfrak{C}_{3}(v) a_{n} b_{n}^{-1} \text { for } x \geq-c_{n} .
\end{aligned}
$$

We now prove (4.3). Note that, as $v>1$,

$$
(1+x)^{v}>1+v x \text { for all } x>0,
$$

which implies that

$$
x-\frac{\left(a_{n} x+b_{n}\right)^{v}-b_{n}^{v}}{2 \lambda^{v}}<0 \text { for all } x>0
$$

since

$$
2 \lambda^{v} x+b_{n}^{v}-\left(a_{n} x+b_{n}\right)^{v}=b_{n}^{v}\left(1+v a_{n} b_{n}^{-1} x-\left(a_{n} b_{n}^{-1} x+1\right)^{v}\right)
$$

by (1.6). By (1.5), (4.10), and the definition of $A_{n}(x)$, we have

$$
\begin{aligned}
A_{n}^{\prime}(x) & =\exp \left\{-n \Psi_{n}(x)+\mathrm{e}^{-x}\right\}\left[-n\left(\Psi_{n}(x)\right)^{\prime}-\mathrm{e}^{-x}\right] \\
& =-A_{n}(x) \mathrm{e}^{-x}\left[1-n a_{n} \mathrm{e}^{x} F_{v}^{\prime}\left(a_{n} x+b_{n}\right)\right] \\
& =-A_{n}(x) \mathrm{e}^{-x}\left[1-\exp \left\{\frac{b_{n}^{v}}{2 \lambda^{v}}\right\} \mathrm{e}^{x} \exp \left\{-\frac{\left(a_{n} x+b_{n}\right)^{v}}{2 \lambda^{v}}\right\}\right] \\
& =-A_{n}(x) e^{-x}\left[1-\exp \left\{x-\frac{\left(a_{n} x+b_{n}\right)^{v}-b_{n}^{v}}{2 \lambda^{v}}\right\}\right] \\
& <0
\end{aligned}
$$

for $x>0$. Noting that $A_{n}(x) \rightarrow 1$ as $x \rightarrow \infty$,

$$
\begin{aligned}
\sup _{x \geq 0}\left|A_{n}(x)-1\right| & =\left|A_{n}(0)-1\right| \\
& =\exp \left\{n r_{v}\left(b_{n}\right)\right\}-1 \\
& \leq n r_{n} \exp \left\{n r_{n}\right\} \\
& <2 \lambda^{v} v^{-1}(v-1) b_{n}^{-v} \exp \left\{\frac{5(v-1)}{2 v \log 2}\right\} \\
& =(v-1) \exp \left\{\frac{5(v-1)}{2 v \log 2}\right\} a_{n} b_{n}^{-1} \\
& =\mathfrak{C}_{4}(v) a_{n} b_{n}^{-1} .
\end{aligned}
$$

The inequalities come from the facts that $\mathrm{e}^{x}-1 \leq x \mathrm{e}^{x}$ for $0 \leq x \leq 1$,

$$
0<n r_{n}\left(b_{n}\right)<2 \lambda^{v} v^{-1}(v-1) b_{n}^{-v}=(v-1) a_{n} b_{n}^{-1},
$$

and

$$
\exp \left\{n r_{n}\left(b_{n}\right)\right\}<\exp \left\{2 \lambda^{v} v^{-1}(v-1) b_{n}^{-v}\right\}<\exp \left\{\frac{2 \lambda^{v} v^{-1}(v-1)}{0.8 \lambda^{v} \log n}\right\}
$$


Combining with (4.9), we have

$$
\sup _{0 \leq x<\infty}\left|F^{n}\left(a_{n} x+b_{n}\right)-\Lambda(x)\right|<\left(\mathfrak{C}_{3}(v)+\mathfrak{C}_{4}(v)\right) a_{n} b_{n}^{-1} .
$$

Secondly, consider the case in which $-c_{n} \leq x<0$. By (1.5), (1.6), and Proposition 3.1, we have

$$
\begin{aligned}
-n \Psi_{n}(x)+\mathrm{e}^{-x} & \\
= & -n\left[\frac{\lambda^{v-1} 2^{-1 / v}}{\Gamma(1 / v)}\left(a_{n} x+b_{n}\right)^{1-v} \exp \left\{-\frac{\left(a_{n} x+b_{n}\right)^{v}}{2 \lambda^{v}}\right\}-r_{v}\left(a_{n} x+b_{n}\right)\right]+\mathrm{e}^{-x} \\
= & -n\left[\frac{\lambda^{v-1} 2^{-1 / v}}{\Gamma(1 / v)}\left(a_{n} x+b_{n}\right)^{1-v} \exp \left\{-\frac{\left(a_{n} x+b_{n}\right)^{v}}{2 \lambda^{v}}\right\}\right. \\
& \left.\quad-\frac{v^{-1}(v-1) \lambda^{2 v-1} 2^{1-1 / v}}{\Gamma(1 / v)}\left(a_{n} x+b_{n}\right)^{1-2 v} d_{n}\left(a_{n} x+b_{n}\right) \exp \left\{-\frac{\left(a_{n} x+b_{n}\right)^{v}}{2 \lambda^{v}}\right\}\right] \\
& +\mathrm{e}^{-x} \\
= & -\left(a_{n} b_{n}^{-1} x+1\right)^{1-v} \exp \left\{-\frac{\left(a_{n} x+b_{n}\right)^{v}-b_{n}^{v}}{2 \lambda^{v}}\right\} \\
& +2 \lambda^{v} v^{-1}(v-1) b_{n}^{-v}\left(a_{n} b_{n}^{-1} x+1\right)^{1-2 v} d_{n}\left(a_{n} x+b_{n}\right) \exp \left\{-\frac{\left(a_{n} x+b_{n}\right)^{v}-b_{n}^{v}}{2 \lambda^{v}}\right\} \\
& +\mathrm{e}^{-x} \\
= & \left(a_{n} b_{n}^{-1} x+1\right)^{1-v} \mathrm{e}^{-x} \\
& \times\left\{-\left[1-2 \lambda^{v} v^{-1}(v-1) b_{n}^{-v}\left(a_{n} b_{n}^{-1} x+1\right)^{-v} d_{n}\left(a_{n} x+b_{n}\right)\right]\right. \\
\quad & \left.\quad \times \exp \left\{-\frac{\left(a_{n} x+b_{n}\right)^{v}-b_{n}^{v}-2 \lambda^{v} x}{2 \lambda^{v}}\right\}+\left(a_{n} b_{n}^{-1} x+1\right)^{v-1}\right\} \\
= & \left(a_{n} b_{n}^{-1} x+1\right)^{1-v} \mathrm{e}^{-x} D_{n}(x),
\end{aligned}
$$

where $0<d_{n}\left(a_{n} x+b_{n}\right)<1$ and

$$
\begin{aligned}
D_{n}(x)=- & \left\{1-2 \lambda^{v} v^{-1}(v-1) b_{n}^{-v}\left(a_{n} b_{n}^{-1} x+1\right)^{-v} d_{n}\left(a_{n} x+b_{n}\right)\right\} \\
& \times \exp \left\{-\frac{\left(a_{n} x+b_{n}\right)^{v}-b_{n}^{v}-2 \lambda^{v} x}{2 \lambda^{v}}\right\}+\left(a_{n} b_{n}^{-1} x+1\right)^{v-1} .
\end{aligned}
$$

Since

$$
a_{n} x+b_{n}>0 \text { for } x>-c_{n}, \quad \mathrm{e}^{-x}>1-x \text { for } x>0,
$$

and

$$
(1+x)^{v}>1-v x \text { and }(1+x)^{-v}<1-v x \text { for }-1<x<0
$$

we have

$$
\begin{aligned}
D_{n}(x)< & -\left(1-\frac{\left(a_{n} x+b_{n}\right)^{v}-b_{n}^{v}-2 \lambda^{v} x}{2 \lambda^{v}}\right) \\
& \times\left\{1-2 \lambda^{v} v^{-1}(v-1) b_{n}^{-v}\left(a_{n} b_{n}^{-1} x+1\right)^{-v} d_{n}\left(a_{n} x+b_{n}\right)\right\}+\left(a_{n} b_{n}^{-1} x+1\right)^{v-1} \\
< & -\left(1-\frac{\left(a_{n} x+b_{n}\right)^{v}-b_{n}^{v}-2 \lambda^{v} x}{2 \lambda^{v}}\right)\left\{1-2 \lambda^{v} v^{-1}(v-1) b_{n}^{-v}\left(1-v a_{n} b_{n}^{-1} x\right)\right\} \\
& +\left(a_{n} b_{n}^{-1} x+1\right)^{v-1}
\end{aligned}
$$




$$
\begin{aligned}
=- & \left\{1-2 \lambda^{v} v^{-1}(v-1) b_{n}^{-v}\left(1-v a_{n} b_{n}^{-1} x\right)\right. \\
& +\left[\left(a_{n} x+b_{n}\right)^{v}-b_{n}^{v}-2 \lambda^{v} x\right] v^{-1}(v-1) b_{n}^{-v}\left(1-v a_{n} b_{n}^{-1} x\right) \\
& \left.\quad-\frac{\left(a_{n} x+b_{n}\right)^{v}-b_{n}^{v}-2 \lambda^{v} x}{2 \lambda^{v}}\right\}+\left(a_{n} b_{n}^{-1} x+1\right)^{v-1} \\
< & -1+2 \lambda^{v} v^{-1}(v-1) b_{n}^{-v}\left(1-v a_{n} b_{n}^{-1} x\right)+\frac{\left(a_{n} x+b_{n}\right)^{v}-b_{n}^{v}-2 \lambda^{v} x}{2 \lambda^{v}} \\
& +\left(a_{n} b_{n}^{-1} x+1\right)^{v-1} \\
< & -1+2 \lambda^{v} v^{-1}(v-1) b_{n}^{-v}\left(1-v a_{n} b_{n}^{-1} x\right)-(v-1) a_{n} b_{n}^{-1} x^{2}+1 \\
< & (v-1) a_{n} b_{n}^{-1}\left[1+v a_{n} b_{n}^{-1} c_{n}\right] \\
< & \left(v^{2}-1\right) a_{n} b_{n}^{-1} .
\end{aligned}
$$

The last inequality follows by (1.6) and (4.6). Meanwhile, by (4.11),

$$
D_{n}(x)>-1+\left(a_{n} b_{n}^{-1} x+1\right)^{v-1}>-(v-1) a_{n} b_{n}^{-1} x .
$$

Hence,

$$
\left|D_{n}(x)\right|<(v-1) a_{n} b_{n}^{-1}(v+1+|x|) .
$$

So, for $n>2$,

$$
\begin{aligned}
\left|-n \Psi_{n}(x)+\mathrm{e}^{-x}\right| & <(v-1)\left(1+a_{n} b_{n}^{-1} x\right)^{1-v} \mathrm{e}^{-x} a_{n} b_{n}^{-1}(v+1+|x|) \\
& <(v-1)\left(1-a_{n} b_{n}^{-1} c_{n}\right)^{1-v} \mathrm{e}^{c_{n}} a_{n} b_{n}^{-1}\left(v+1+c_{n}\right) \\
& <\mathfrak{C}_{5}(v) .
\end{aligned}
$$

Thus,

$$
\begin{aligned}
\Lambda(x) \mid & A_{n}(x)-1 \mid \\
& =\Lambda(x)\left|\exp \left\{-n \Psi_{n}(x)+\mathrm{e}^{-x}\right\}-1\right| \\
& <\Lambda(x) \exp \left\{\left(-n \Psi_{n}(x)+\mathrm{e}^{-x}\right) \theta\right\}\left|-n \Psi_{n}(x)+\mathrm{e}^{-x}\right| \\
& <(v-1) \exp \left\{\mathfrak{C}_{5}(v)\right\}\left(1-a_{n} b_{n}^{-1} c_{n}\right)^{1-v} a_{n} b_{n}^{-1} \sup _{-c_{n} \leq x<0}\left\{(v+1+|x|) \mathrm{e}^{-x} \Lambda(x)\right\} \\
& <\mathfrak{C}_{6}(v) a_{n} b_{n}^{-1} .
\end{aligned}
$$

Now combining this with (4.8) and (4.9), we complete the proof of (4.4).

Finally, consider the case in which $-\infty<x<-c_{n}$. Note that

$$
\Lambda(x) \leq \Lambda\left(-c_{n}\right)=\frac{v}{2 \lambda^{v}} a_{n} b_{n}^{-1}
$$

and

$$
\begin{aligned}
\sup _{x \leq-c_{n}}\left|F^{n}\left(a_{n} x+b_{n}\right)-\Lambda(x)\right| & <F^{n}\left(b_{n}-a_{n} c_{n}\right)+\Lambda\left(-c_{n}\right) \\
& <\sup _{x \in\left[-c_{n}, 0\right)}\left|F^{n}\left(a_{n} x+b_{n}\right)-\Lambda(x)\right|+2 \Lambda\left(-c_{n}\right) \\
& <\left(\mathfrak{C}_{3}(v)+\mathfrak{C}_{6}(v)\right) a_{n} b_{n}^{-1}+\frac{v}{\lambda^{v}} a_{n} b_{n}^{-1} \\
& =\mathfrak{C}_{7}(v) a_{n} b_{n}^{-1} .
\end{aligned}
$$

This completes the proof of (4.5). The proof of Theorem 2.1 is complete. 


\section{Acknowledgements}

The authors would like to thank the Editor and the anonymous referee for careful readings and for their comments which greatly improved the paper.

\section{References}

Anderson, C. W. (1971). Contributions to the asymptotic theory of extreme values. Doctoral Thesis, University of London.

Balkema, A. A. And de HaAn, L. (1990). A convergence rate in extreme-value theory. J. Appl. Prob. 27, 577-585.

CAStro, L. C. E. (1987). Uniform rate of convergence in extreme-value theory: normal and gamma models. Ann. Sci. Univ. Clermont-Ferrand II, Prob. Appl. 6, 25-41.

Cheng, S. H., De HaAn, L. And Huang, X. (1997). Rate of convergence of intermediate order statistics. J. Theoret. Prob. 1, 1-23.

Cohen, J. P. (1982a). Convergence rates for the ultimate and penultimate approximations in extreme-value theory. Adv. Appl. Prob. 14, 833-854.

Cohen, J. P. (1982b). The penultimate form of approximation to normal extremes. Adv. Appl. Prob. 14, 324-339.

De HAAN, L. AND Resnick, S. I. (1996). Second-order regular variation and rates of convergence in extreme-value theory. Ann. Prob. 24, 97-124.

FALK, A. (1986). Rates of uniform convergence of extreme order statistics. Ann. Inst. Statist. Math. 38, $245-262$.

Gomes, M. I. (1984). Penultimate limiting forms in extreme value theory. Ann. Inst. Statist. Math. 36, 71-85.

Gomes, M. I. AND DE HAAN, L. (1999). Approximatation by penultimate extreme value distributions. Extremes 2 , $71-85$.

Hall, P. (1979). On the rate of convergence of normal extremes. J. Appl. Prob. 16, 433-439.

Hall, W. J. and Wellner, J. A. (1979). The rate of convergence in law of the maximum of an exponential sample. Statist. Neerlandica 33, 151-154.

Kaufmann, E. (1995). Von Mises conditions, $\delta$-neighborhoods and rates of convergence for maxima. Statist. Prob. Lett. 25, 63-70.

Kaufmann, E. (2000). Penultimate approximations in extreme value theory. Extremes 3, 39-55.

LeAdbetter, M. R., Lindgren, G. And Rootzén, H. (1983). Extremes and Related Properties of Random Sequences and Processes. Springer, New York.

Nelson, D. B. (1991). Conditional heteroskedasticity in asset returns: a new approach. Econometrica 59, 347-370.

Peng Z., Tong B. And Nadarajah, S. (2009). Tail behavior of the general error distribution. Commun. Statist. Theory Meth. 38, 1884-1892.

REISs, R.-D. (1989). Approximate Distributions of Order Statistics. Springer, New York.

Resnick, S. I. (1987). Extreme Values, Regular Variation and Point Processes. Springer, New York.

Rootzén, H. (1983). The rate of convergence of extremes of stationary normal sequences. Adv. Appl. Prob. 15, 54-80.

Smith, R. L. (1982). Uniform rates of convergence in extreme-value theory. Adv. Appl. Prob. 14, 600-622. 